\title{
Cooperative Vehicle-Infrastructure Localization based on the Symmetric Measurement Equation Filter
}

\author{
Feihu Zhang ${ }^{\mathrm{a}}$, Gereon Hinz ${ }^{\mathrm{a}}$, Dhiraj Gulati ${ }^{\mathrm{a}}$, Daniel Clarke ${ }^{\mathrm{b}}$, Alois Knoll ${ }^{\mathrm{c}}$ \\ ${ }^{a}$ fortiss GmbH, Guerickestr. 25, 80805 München, Germany \\ ${ }^{b}$ Cranfield University, United Kingdom \\ ${ }^{c}$ Institute of Robotics and Embedded Systems, Technische Universität München, 85748 Garching \\ bei München, Germany
}

\begin{abstract}
Precise and accurate localization is important for safe autonomous driving. Given a traffic scenario which has multiple vehicles equipped with internal sensors for self-localization, and external sensors from the infrastructure for vehicle localization, vehicle-infrastructure communication can be used to improve the accuracy and precision of localization. However, as the number of vehicles in a scenario increases, associating measurement data with the correct source becomes increasingly challenging. We propose a solution utilizing the symmetric measurement equation filter (SME) for cooperative localization to address data association issue, as it does not require an enumeration of measurement-to-target associations. The principal idea is to define a symmetrical transformation which maps measurements to a homogeneous function, thereby effectively addressing several challenges in vehicle-infrastructure scenarios such as data association, bandwidth limitations and registration/configuration of the external sensor. To the best of our knowledge, the proposed solution is among the first to address all these issues of cooperative localization simultaneously, by utilizing the topology information of the vehicles.
\end{abstract}

Keywords: Symmetric Measurement Equation (SME) filter; data association.

\footnotetext{
*Corresponding author. Tel.: +49 89360352239.

Email address: zhangefortiss.org (Feihu Zhang)
} 


\section{Introduction}

Over the last years, vehicle-to-vehicle and vehicle-to-infrastructure localization has evolved as an important topic in the intelligent transportation domain [1]. Cooperative localization has a number of benefits ranging from improved precision to improved situational awareness for applications such as monitoring traffic flow. In modern transportation systems, the situational awareness system depends on the localization uncertainty. It is possible to guide vehicles with individual optimal routes, with the help of low precision localization information calculated in the cloud center. However, for safety applications, high precision localization information is utilized to assist other advanced driver assistance systems (ADAS). For instance, precise localization information helps the lane departure system keep the vehicle in its trajectory when there is a maneuvering behavior.

The commonly available Global Positioning System (GPS) provides an accurate estimate of receiver location and is widely used by the automotive domain. But the signal characteristics mean that its integrity is susceptible to interference (intentional or otherwise) and the receiving antenna should have a clear view of the sky (e.g. [2]). This becomes problematic in urban environments with high buildings, tunnels and parking lots. Therefore improved methodologies utilize sensors which are both internal and external to the vehicle [3], [4].

Furthermore, with the development of Car-2-Car (C2C) and Car-2-Infrastructure (C2I) communication techniques, sharing information, such as sensor measurements and state estimates, across the whole network has become possible [5]. These new communication networks can be utilized to improve the perception performance, as cooperative localization can lead to better state estimates than separate self-localization by each individual vehicle [1]. Many methods have been proposed for the vehicle-infrastructure cooperative localization, e.g. Extended Kalman Filter [6], Markov Localization [7], Maximum Likelihood Estimation [8] and Maximum A Posteriori Estimation [9]. But a number of challenges still exist:

- The data association challenge.

One of the most important tasks for cooperative localization is that of Data Association, in which sensor measurements are correctly associated to their corresponding targets in order to estimate the physical state. The development of C2C and C2I techniques supports vehicles in localizing and identifying other traffic participants correctly. However, in case of an uncertain (or even missing) measurement-to-target association within the network, it is a significant challenge 
to correctly compute a bias free state estimate. Also, measurements are often obscured or cluttered (measurements not only originate from interesting targets, but also from the outliers), further diluting the strength of association and increasing the localization uncertainty.

- The bandwidth challenge.

A cooperative localization system requires that each node transmits both state and covariance estimations. System which contains a multiple dimensional state representation (position, velocity, acceleration) with a high update frequency implies a significant amount of data. The full covariance matrix (the number of dimensions squared) is required to fully characterize the multiple dimensional variation. This in turn requires a high bandwidth multiplexed signal carrier and as the number of vehicles increases, the network is likely to get overloaded and thus unusable.

- The coordinate transformation challenge.

Coordinate transformation plays an important role in cooperative localization. Measurements are acquired from internal and external sensors to localize the positions. The internal sensor only provides the absolute location in 2D global coordinates whereas the external sensor often provides the relative position in $2 \mathrm{D}$ local coordinates. But in a dynamic environment where the configuration of the external sensor is unknown, the relative transformation between different coordinate frames increases the localization issues.

A methodology for vehicle-infrastructure cooperative localization based on the Symmetric Measurement Equation (SME) filter [10] is proposed, which extends the previous work of [11]. With the SME filter, a new type of symmetrical measurement transformation based on homogeneous symmetric functions has been introduced [12]. The key idea is to convert measurement data with unknown association into a symmetric measurement equation to estimate the corresponding states [13].

The work-flow of the proposed SME filter is as follows: Measurements from both internal and external sensors are projected to a symmetric equation to acquire new observations, where the SME filter recursively estimates the dynamic states.

The advantages of the SME filter are as follows:

Firstly, the data association challenge is addressed. The SME filter provides a new solution to avoid the data association by using a symmetric measurement 


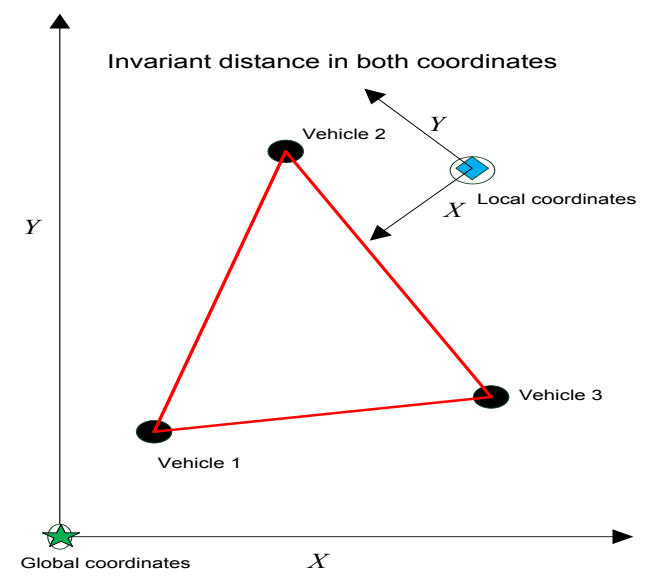

Figure 1: Topology of cooperative localization system

equation to build up a pseudo-measurement space in which data association is unnecessary.

Secondly, the bandwidth challenge is addressed. Since the SME filter is a recursive centralized Bayes filter which requires the network to transmit only observations, the amount of data that needs to be exchanged is reduced. In contrast to other methods, the proposed approach has the minimal bandwidth requirement.

Thirdly, the coordinate transformation challenge is addressed. Measurements are converted to a symmetric measurement equation based on homogeneous symmetric functions, which avoids the transformation between different coordinate system. Even if the configuration of the external sensor is unknown, the proposed SME filter still works.

This paper is structured as follows: Sec. 2 briefly describes the scenario of the vehicle-infrastructure cooperative localization. Sec. 3 introduces more details about the SME filter with the implementation details. Sec. 4 presents simulation results. Finally, the paper is concluded in Sec. 5.

\section{Background description}

Fig. 1 illustrates the vehicle-infrastructure cooperative localization scenario and is described as follows:

- Each vehicle is able to localize itself according to an absolute reference. 
Here we assume that the measurements given by internal sensors (e.q. onboard GPS) are in a 2D global coordinate system. Further values in the 2D global coordinate system represent location of the vehicles in Cartesian coordinate system.

- The infrastructure is able to measure the relative position of the vehicles. Here we assume that the measurements given by external sensors are in a $2 \mathrm{D}$ local coordinate system. Further values in the 2D local coordinate system represent the relative location of the vehicles with respect to the external sensor in Cartesian coordinate system.

- A communication network, to exchange information between the cars and the infrastructure, is available. Here we assume that there is no delay in the data-link and no clutter exists, e.g. there is no false detection in the scenario.

- The communication method and protocol are not used to identify the individual vehicles. Also, there is no prior information regarding to the configuration of the infrastructure, i.e., the location and orientation of the external sensor are unknown.

Assuming that the internal sensors provide measurements with large uncertainties, the localization becomes imprecise. However, by cooperative localization, the precision is ensured with the help of the external sensor since its measurements are more precise [1].

Much work has been done for cooperative localization: centralized solution [6][14] and decentralized solution [15, 16, 17]. In the centralized solution, all vehicles are considered as a single system where the estimation is computed using the Kalman filter. There is no need of high communication bandwidth because covariance of the state is not transmitted. But as the number of vehicles increases, the data association computation grows exponentially.

In contrast to the centralized architecture, a decentralized solution uses multiple fusion centers. Each fusion center handles part of the local information (only the observed neighbors). Still, the computational demand is very high. Moreover, it often exceeds the network bandwidth limitations since each fusion center requires both the states and the corresponding covariances. Therefore, both the communication and computational demands present a number of difficulties in decentralized solutions.

None of the above solution considers the coordinate transformation issue during the localization process. Both centralized and decentralized approaches assume that the transformation between the global measurement and the relative 
measurement is known, or at least could be estimated with the associated measurements. Cooperative localization only works under the condition that all measurements are processed within a known coordinate system. On the other hand, in highly dynamic environments, the configuration of the external sensor is difficult to estimate due to the data association challenge.

To the best of our knowledge, it is still difficult to address the aforementioned challenges simultaneously. In the next section, the SME filter is presented which takes into account all the issues. This work is developed in cooperation with the SADA Project (BMWi funded, 'IKT für Elektromobilität III' [18]), to evaluate the performance of the cooperative localization between vehicle and infrastructure sensors.

\section{The Symmetric Measurement Equation (SME) filter}

The SME filter based on homogeneous symmetric function is proposed because of its ability to simultaneously address the identified issues within a multitarget tracking scenario.

\subsection{Overview on SME filter}

A major hurdle in multi-target tracking domain is the data association between the measurement and either a new or an existing track. In the past decades, various methods have been developed to address this such as the Joint Probabilistic Data Association filter (JPDA) [19], the Probability Hypothesis Density filter (PHD) [20] and the Multi Hypothesis Tracking filter [21] (MHT). However, as the number of targets grows, the computational requirements grow exponentially.

The Symmetric Measurement Equation (SME) filter removes the data association by utilizing a symmetrical transformation. This allows us to bypass the combinatorial complexity of the association tasks. The SME filter transforms the association issue into a nonlinear state estimation problem with non-additive Gaussian noise. In this way, one difficult problem is traded for another difficult, but different, problem [22].

The first work on the SME filter was presented by Kamen [10]. It was also proposed to address the nonlinear conditions by the Unscented Kalman filter [22] and the Particle filter [23].

M. Baum [24] implemented the SME filter in the field of group targets tracking. The result illustrates that the SME filter is an effective solution for the multiple target tracking. In addition, it was shown that the SME filter is suitable for a large number of closely-spaced targets during the tracking phase. 
This paper applies the SME filter to vehicle-infrastructure cooperative localization. Assuming there is no missed or false detection, the SME filter is utilized to localize the vehicles based on the symmetric measurement equations.

\subsection{Mathematic Background of the SME Filter}

The idea of the SME filter is to generate 'pseudo-measurements' that consist of symmetric functions of the original measurement from targets.

\section{- Problem formulation}

For $n$ dimension target state $\underline{x}_{k}^{1}, \cdots, \underline{x}_{k}^{N}$, where $k$ denotes the step and $N$ is the number of the targets, the joint set-valued state is represented as $\mathbf{x}_{k}=$ $\left[\left(\underline{x}_{k}^{1}\right)^{\mathrm{T}}, \cdots,\left(\underline{x}_{k}^{N}\right)^{\mathrm{T}}\right]^{\mathrm{T}} \in \mathbb{R}^{\mathrm{n} \cdot \mathrm{N}}$.

With the same manner, $\mathbf{y}_{k}=\left[\left(h_{k}^{1}\left(\mathbf{x}_{k}\right)\right)^{\mathrm{T}},\left(h_{k}^{2}\left(\mathbf{x}_{k}\right)\right)^{\mathrm{T}}, \cdots,\left(h_{k}^{N}\left(\mathbf{x}_{k}\right)\right)^{\mathrm{T}}\right]^{\mathrm{T}} \in \mathbb{R}^{\mathrm{m} \cdot \mathrm{N}}$ denotes the set-valued measurement, where $m$ is the dimension of measurement function $h_{k}^{i}\left(\mathbf{x}_{k}\right)$.

To avoid the data association between the measurements and targets, each component $h_{k}^{i}$ should remain unchanged for any permutation in the argument of the states, which is called 'symmetric transformation'.

\subsubsection{Measurement Model}

Assuming at each step the measurements are available, the following equations give the 'pseudo-measurements'

$$
\begin{gathered}
\underline{y}_{k}^{1}=h_{k}^{1}\left(\underline{x}_{k}^{1}+v_{k}^{1}, \underline{x}_{k}^{2}+v_{k}^{2}, \cdots, \underline{x}_{k}^{N}+v_{k}^{N}\right) \\
\vdots \\
\underline{y}_{k}^{N}=h_{k}^{N}\left(\underline{x}_{k}^{1}+v_{k}^{1}, \underline{x}_{k}^{2}+v_{k}^{2}, \cdots, \underline{x}_{k}^{N}+v_{k}^{N}\right)
\end{gathered}
$$

where each $h_{k}^{i}$ is a permutation in the symmetric group which specifies the unknown association assignment and $v_{k}^{i}$ is considered as the additive zero-mean white noise. Combined with the joint set-valued state, equation (1) can be composed as following

$$
\underbrace{\left[\begin{array}{c}
y_{k}^{1} \\
\vdots \\
y_{k}^{N}
\end{array}\right]}_{\mathbf{y}_{k}}=\underbrace{\left[\begin{array}{c}
h_{k}^{1}\left(\mathbf{x}_{k}, \mathbf{v}_{k}\right) \\
\vdots \\
h_{k}^{N}\left(\mathbf{x}_{k}, \mathbf{v}_{k}\right)
\end{array}\right]}_{\mathbf{H}_{k}\left(\mathbf{x a}_{k}\right)}
$$

where $\mathbf{y}_{k}$ denotes the joint set-valued measurement in the SME filter. 


\subsubsection{Process Model}

The target system model in SME filter is represented as

$$
\begin{gathered}
\underline{x}_{k+1}^{1}=A_{k}^{1} \underline{x}_{k}^{1}+w_{k}^{1} \\
\vdots \\
\underline{x}_{k+1}^{N}=A_{k}^{N} \underline{x}_{k}^{N}+w_{k}^{N}
\end{gathered}
$$

where $A_{k}$ is the process transformation and $w_{k}$ is the additive white noise. Equation (3) can also be composed as

$$
\underbrace{\left[\begin{array}{c}
\underline{x}_{k+1}^{1} \\
\vdots \\
\underline{x}_{k+1}^{N}
\end{array}\right]}_{\mathbf{x}_{k+1}}=\underbrace{\left[\begin{array}{lll}
A_{k}^{1} & & \\
& \ddots & \\
& & A_{k}^{N}
\end{array}\right]}_{\mathbf{A}_{k}} \cdot \underbrace{\left[\begin{array}{c}
\underline{x}_{k}^{1} \\
\vdots \\
\underline{x}_{k}^{N}
\end{array}\right]}_{\mathbf{x}_{k}}+\underbrace{\left[\begin{array}{c}
w_{k}^{1} \\
\vdots \\
w_{k}^{N}
\end{array}\right]}_{\mathbf{w}_{k}}
$$

\subsubsection{Symmetric Transformation}

Since the SME filter removes the data association between the measurements and the targets, a symmetrical transformation is thus required.

The transformation is done by adding or multiplying acquired measurements such that all generated 'pseudo-measurements' have values from all the targets. This makes the measurement matrix $\mathbf{H}_{k}\left(\mathbf{x}_{k}\right)$ independent of the permutation in the argument of the state $\mathbf{x}_{k}$. Thus there is no data association.

Two simple examples of how to construct the symmetric measurement equations for three targets are given as follows:

Example 1. Sum-of-product:

$$
S_{\text {prod }}=\left[\begin{array}{c}
m_{1}+m_{2}+m_{3} \\
m_{1} m_{2}+m_{2} m_{3}+m_{1} m_{3} \\
m_{1} m_{2} m_{3}
\end{array}\right]
$$

\section{Example 2. Sum-of-powers:}

$$
S_{\text {pow }}=\left[\begin{array}{c}
m_{1}+m_{2}+m_{3} \\
m_{1}^{2}+m_{2}^{2}+m_{3}^{2} \\
m_{1}^{3}+m_{2}^{3}+m_{3}^{3}
\end{array}\right]
$$

For three targets that evolve according to a random walk model in a one dimension scenario, the process model and the measurement model are thus given by one-dimension identity matrix $\mathrm{I}_{1} . m_{i}$ is the measurement from the $i_{t h}$ target. 
If the measurement-to-target association is known, the Kalman filter is applied individually as following:

$$
\begin{gathered}
\underline{x}_{k+1}^{i}=\underline{x}_{k}^{i}+w_{k}^{i} \\
m_{i}=\underline{x}_{k}^{i}+v_{k}^{i}, i=1,2,3 .
\end{gathered}
$$

where $w_{k}^{i}$ and $v_{k}^{i}$ are Gaussian white noises. With the same manner, it can also be represented as

$$
\begin{gathered}
\underbrace{\left[\begin{array}{l}
\underline{x}_{k+1}^{1} \\
\underline{x}_{k+1}^{2} \\
\underline{x}_{k+1}^{3}
\end{array}\right]}_{\mathbf{x}_{k+1}}=\underbrace{\left[\begin{array}{c}
\underline{x}_{k}^{1} \\
\underline{x}_{k}^{2} \\
\underline{x}_{k}^{3}
\end{array}\right]}_{\mathbf{x}_{k}}+\underbrace{\left[\begin{array}{c}
w_{k}^{1} \\
w_{k}^{2} \\
w_{k}^{3}
\end{array}\right]}_{\mathbf{w}_{k}} \\
\underbrace{\left[\begin{array}{c}
\underline{y}_{k}^{1} \\
\underline{y}_{k}^{2}
\end{array}\right]}_{\mathbf{y}_{k}}=\left[\begin{array}{c}
m_{1} \\
m_{2}^{2} \\
m_{3}^{3}
\end{array}\right]=\underbrace{\left[\begin{array}{lll}
1 & 0 & 0 \\
0 & 1 & 0 \\
0 & 0 & 1
\end{array}\right]}_{\mathbf{H}_{k}} \cdot \underbrace{\left[\begin{array}{c}
\underline{x}_{k}^{1} \\
\underline{x}_{k}^{2} \\
\underline{x}_{k}^{3}
\end{array}\right]}_{\mathbf{x}_{k}}+\underbrace{\left[\begin{array}{c}
v_{k}^{1} \\
v_{k}^{2} \\
v_{k}^{3}
\end{array}\right]}_{\mathbf{v}_{k}}=\underbrace{\left[\begin{array}{c}
h_{k}^{1}\left(\mathbf{x}_{k}, \mathbf{v}_{k}\right) \\
h_{k}^{2}\left(\mathbf{x}_{k}, \mathbf{v}_{k}\right) \\
h_{k}^{3}\left(\mathbf{x}_{k}, \mathbf{v}_{k}\right)
\end{array}\right]}_{\mathbf{H}_{k}\left(\mathbf{x}_{k}, \mathbf{v}_{k}\right)}
\end{gathered}
$$

It is observed that each component $h_{k}^{i}$ depends on the permutation order in setvalued state $\mathbf{x}_{k}$, which is called 'data association'.

If the measurement-to-target association is unknown, the performance of Eq. (8) drops immediately. The SME filter when applied, makes $h_{k}^{i}$ remain unchanged for any permutation in the argument of the state, which is called 'symmetric transformation function':

$$
\begin{gathered}
\underbrace{\left[\begin{array}{l}
\underline{x}_{k+1}^{1} \\
\underline{x}_{k+1}^{2} \\
\underline{x}_{k+1}^{3}
\end{array}\right]}_{\mathbf{x}_{k+1}}=\underbrace{\left[\begin{array}{l}
\underline{x}_{k}^{1} \\
\underline{x}_{k}^{2} \\
\underline{x}_{k}^{3}
\end{array}\right]}_{\mathbf{x}_{k}}+\underbrace{\left[\begin{array}{c}
w_{k}^{1} \\
w_{k}^{2} \\
w_{k}^{3}
\end{array}\right]}_{\mathbf{w}_{k}} \\
\underbrace{\left[\begin{array}{c}
\underline{y}_{k}^{1} \\
\underline{y}_{k}^{2} \\
\underline{y}_{k}^{3}
\end{array}\right]}_{\mathbf{y}_{k}}=\left[\begin{array}{c}
m_{1}+m_{2}+m_{3} \\
m_{1}^{2}+m_{2}^{2}+m_{3}^{2} \\
m_{1}^{3}+m_{2}^{3}+m_{3}^{3}
\end{array}\right]=\underbrace{\left[\begin{array}{c}
h_{k}^{1}\left(\mathbf{x}_{k}, \mathbf{v}_{k}\right) \\
h_{k}^{2}\left(\mathbf{x}_{k}, \mathbf{v}_{k}\right) \\
h_{k}^{3}\left(\mathbf{x}_{k}, \mathbf{v}_{k}\right)
\end{array}\right]}_{S_{p o w}=\mathbf{H}_{k}\left(\mathbf{x}_{k}\right)}
\end{gathered}
$$

Although the pseudo-measurement $S$ (Sum-of-powers) represents the information from a linear space to a nonlinear space, the original measurement $m_{i}$ can still be recovered uniquely. Therefore, there is no information loss with the symmetrical transformation. Since the transformed equations are symmetrically represented which does not rely on the permutation order in $\mathbf{x}_{k}$, the data association 
issue is thus addressed. By using nonlinear Bayesian estimators such as Extended Kalman filter (EKF) [10], Unscented Kalman filter (UKF) [22] or Particle filter (PF) [23], the set-valued states $\mathbf{x}_{k}$ is estimated recursively.

\subsection{Implementation of SME filter}

The mathematic background of the SME filter has been briefly introduced in Sec. 3.2. However, there are still open issues regarding to the implementation, e.g. how to utilize the SME filter in vehicle-infrastructure cooperative localization? How to utilize external sensor in unknown environments?

For the process model, each single state $\underline{x}_{k}^{i}=\left[p_{x, k}^{i}, \dot{p}_{x, k}^{i}, p_{y, k}^{i}, \dot{p}_{y, k}^{i}\right]^{T}$ consists of the positions $\left(p_{x, k}, p_{y, k}\right)$ and velocities $\left(\dot{p}_{x, k}, \dot{p}_{y, k}\right)$, where the set-valued state is thus given by $\mathbf{x}_{k}=\left[\left(\underline{x}_{k}^{1}\right)^{\mathrm{T}}, \cdots,\left(\underline{x}_{k}^{N}\right)^{\mathrm{T}}\right]^{\mathrm{T}}$.

Following the constant velocity model, the related parameters can be represented as:

$$
\begin{aligned}
& A_{k}^{1}=A_{k}^{2}=\cdots=A_{k}^{N}=\left[\begin{array}{cccc}
1 & T & 0 & 0 \\
0 & 1 & 0 & 0 \\
0 & 0 & 1 & T \\
0 & 0 & 0 & 1
\end{array}\right] \\
& \mathbf{A}_{k}=\left[\begin{array}{lll}
A_{k}^{1} & & \\
& \ddots & \\
& & A_{k}^{N}
\end{array}\right] \\
& Q_{k}^{1}=\cdots=Q_{k}^{N}=\delta^{2}\left[\begin{array}{cccc}
T^{2} / 4 & T^{2} / 2 & 0 & 0 \\
T^{2} / 2 & T & 0 & 0 \\
0 & 0 & T^{2} / 4 & T^{2} / 2 \\
0 & 0 & T^{2} / 2 & T
\end{array}\right] \\
& \mathbf{Q}_{k}=\left[\begin{array}{lll}
Q_{k}^{1} & & \\
& \ddots & \\
& & Q_{k}^{N}
\end{array}\right]
\end{aligned}
$$

In constant turn rate model, the related parameters are changed to

$$
A_{k}^{1}=A_{k}^{2}=\cdots=A_{k}^{N}=\left[\begin{array}{cccc}
1 & \frac{\sin \omega T}{\omega} & 0 & -\frac{1-\cos \omega T}{\omega} \\
0 & \cos \omega T & 0 & -\sin \omega T \\
0 & \frac{1-\cos \omega T}{\omega} & 1 & \frac{\sin \omega T}{\omega} \\
0 & \sin \omega T & 0 & \cos \omega T
\end{array}\right]
$$




$$
\begin{aligned}
& \mathbf{A}_{k}=\left[\begin{array}{lll}
A_{k}^{1} & & \\
& \ddots & \\
& & A_{k}^{N}
\end{array}\right] \\
& Q_{k}^{i}=\delta^{2}\left[\begin{array}{cccc}
\frac{2(\omega T-\sin \omega T)}{\omega^{3}} & \frac{1-\cos \omega T}{\omega^{2}} & 0 & \frac{\omega T-\sin \omega T}{\omega^{2}} \\
\frac{1-\cos \omega T}{\omega^{2}} & T & \frac{-(\omega T-\sin \omega T)}{\omega^{2}} & 0 \\
0 & \frac{-(\omega T-\sin \omega T)}{\omega^{2}} & \frac{2(\omega T-\sin \omega T)}{\omega^{3}} & \frac{1-\cos \omega T}{\omega^{2}} \\
\frac{\omega T-\sin \omega T}{\omega^{2}} & 0 & \frac{1-\cos \omega T}{\omega^{2}} & T
\end{array}\right] \text {, } \\
& \mathbf{Q}_{k}=\left[\begin{array}{lll}
Q_{k}^{1} & & \\
& \ddots & \\
& & Q_{k}^{N}
\end{array}\right]
\end{aligned}
$$

The process model for set-valued state is thus given as:

$$
\mathbf{x}_{k+1}=\mathbf{A}_{k} \cdot \mathbf{x}_{k}+\mathbf{w}_{k}
$$

where $\mathbf{Q}_{k}$ is denoted as the covariance of the process noise $\mathbf{w}_{k}$, $\mathbf{T}$ is the sampling interval and $\delta$ is the standard deviation. For constant turn rate scenarios, $\omega$ is considered as the angular (turn) rate in circular motion. More details can be found in [25].

In measurement model, the transformation of the original measurements into the symmetric equation form is done both for the internal and the external sensors.

To map the state to the observation space, measurements from internal sensors are converted as the Sum-of-powers:

$$
\mathbf{y I}_{k}=\left[\underline{\mathbf{y x}}_{k}, \underline{\mathbf{y y}}_{k}\right]^{T}
$$

where

$$
\begin{aligned}
& \underline{\mathbf{y x}}_{k}=\left[\sum_{i=1}^{N} p_{x, k}^{i}, \sum_{i=1}^{N}\left(p_{x, k}^{i}\right)^{2}, \cdots, \sum_{i=1}^{N}\left(p_{x, k}^{i}\right)^{N}\right]^{T} \\
& \underline{\mathbf{y y}}_{k}=\left[\sum_{i=1}^{N} p_{y, k}^{i}, \sum_{i=1}^{N}\left(p_{y, k}^{i}\right)^{2}, \cdots, \sum_{i=1}^{N}\left(p_{y, k}^{i}\right)^{N}\right]^{T}
\end{aligned}
$$

The coordinate transformation for external sensor is unknown, the topology information (distance between vehicles) is therefore utilized as follows: 


$$
\mathbf{y E}_{k}=\left[\sum_{i=1}^{N-1} \sum_{j=i+1}^{N}\left(p_{x, k}^{i}-p_{x, k}^{j}\right)^{2}+\sum_{i=1}^{N-1} \sum_{j=i+1}^{N}\left(p_{y, k}^{i}-p_{y, k}^{j}\right)^{2}\right]
$$

No matter in which coordinate system, the global or the local, the relative distances between the vehicles are invariant. Equation (16) is thus considered as an additional measurement from the external sensor, even when the coordinate transformation is unknown. In this way, more precise information from external sensor is also utilized in the SME filter.

The final pseudo-measurement $\mathbf{y}_{k}$ is consisted as

$$
\mathbf{H}_{k}=\left[\begin{array}{c}
\mathbf{y} \mathbf{I}_{k} \\
\mathbf{y} \mathbf{E}_{k}
\end{array}\right]=\left[\begin{array}{c}
p_{x, k}^{1}+p_{x, k}^{2}+, \cdots,+p_{x, k}^{N} \\
\left(p_{x, k}^{1}\right)^{2}+\left(p_{x, k}^{2}\right)^{2}+, \cdots,+\left(p_{x, k}^{N}\right)^{2} \\
\vdots \\
\left(p_{x, k}^{1}\right)^{N}+\left(p_{x, k}^{2}\right)^{N}+, \cdots,+\left(p_{x, k}^{N}\right)^{N} \\
p_{y, k}^{1}+p_{y, k}^{2}+, \cdots,+p_{y, k}^{N} \\
\left(p_{y, k}^{1}\right)^{2}+\left(p_{y, k}^{2}\right)^{2}+, \cdots,+\left(p_{y, k}^{N}\right)^{2} \\
\vdots \\
\left(p_{x, k}^{1}\right)^{N}+\left(p_{x, k}^{2}\right)^{N}+, \cdots,+\left(p_{x, k}^{N}\right)^{N} \\
\sum_{i=1}^{N-1} \sum_{j=i+1}^{N}\left(p_{x, k}^{i}-p_{x, k}^{j}\right)^{2}+\sum_{i=1}^{N-1} \sum_{j=i+1}^{N}\left(p_{y, k}^{i}-p_{y, k}^{j}\right)^{2}
\end{array}\right]
$$

Based on the above procedure, multiple challenges in vehicle-infrastructure cooperative localization are addressed simultaneously. The state is estimated by the pseudo-measurement in consecutive frames. We would like to remind the reader that the new measurement noise covariance matrix $\mathbf{R}_{k}$ is calculated with respect to the SME pseudo-measurement space, not in the original Cartesian measurement space. More details of the covariance matrix $\mathbf{R}_{k}$ can be found in $[12,22]$. We do not consider this problem further in this paper. Compared to the previous work in $[10,12,22]$, the proposed solution utilizes target topology information as an additional measurement during the tracking phase. Measurements from both internal and external sensors are collected as a set-value measurement to update states of all the vehicles, given the configuration of the external sensor is unknown.

Also, there is no difference between sum of power, or sum of product. The goal of the symmetric measurement equation is to transfer the original measurement from linear space to the nonlinear space where the data association issue is avoided. The performance should remain the same with different representations, 
since the original measurement is recovered uniquely from both transformed functions. Based on the symmetric non-linear transformation equation, the SME filter can be implemented by the non-linear Bayesian state estimators such as Extended Kalman filter, Unscented Kalman filter and the Particle filter. In this paper, the particle filter is utilized to execute the SME filter with the sum of powers format.

\section{Simulation and discussion}

\subsection{Simulation}

The simulation was implemented with five vehicles on the two dimension ground plane. The performance of the SME filter is demonstrated with respect to the Kalman filter and the Gaussian Mixture Probability Hypothesis Density (GMPHD) filter. The evaluated scenarios are: Constant Velocity (CV) model and Constant Turn Maneuver (CTM) model.

In simulation, vehicles are equipped with internal sensors to measure their global coordinates. The external sensor provides observations with a higher precision in a local coordinate system where the transformation to the global coordinate system is unknown. Such information is thus unnecessary for the filtering phase in both the Kalman filter and the PHD filter.

The simulation is based on the following assumptions:

- Each vehicle gives rise to exactly one single measurement per sensor, i.e., no missed detection.

- There are no false detections during the whole process, i.e., measurements originate from vehicles.

- It is not able to identify the others through the communication system, i.e., the measurement-to-target association is unknown.

During the process, noises from internal sensors are assumed to be white Gaussian distributed with zero mean and covariance diag $[0.5,0.5]$, and the noise from external sensor has zero mean and covariance diag $[0.1,0.1]$. The initial states for the five vehicles are as following: $\underline{x}_{1}=[0,-1.5,100,-1]^{\mathrm{T}}, \underline{x}_{2}=$ $[0,-1.5,0,1]^{\mathrm{T}}, \underline{x}_{3}=[0,0,0,3]^{\mathrm{T}}, \underline{x}_{4}=[0,1.5,0,1]^{\mathrm{T}}, \underline{x}_{5}=[0,1.5,100,-1]^{\mathrm{T}}$. Thus the joint state for the SME filter is given by $\mathrm{x}=\left[\underline{x}_{1}, \underline{x}_{2}, \underline{x}_{3}, \underline{x}_{4}, \underline{x}_{5}\right]^{\mathrm{T}}$.

The corresponding process model $\mathbf{A}$ in the SME filter has already been introduced in Sec. 3.3 in Eq. (4), where the sampling interval $\mathrm{T}$ is defined as 1. 
The standard deviation of the process noise $\delta$ is defined as 0.5 and the process covariance matrix $\mathrm{Q}$ is initialed as $\operatorname{diag}[2.5,1,2.5,1, \cdots, 2.5,1,2.5,1]$.

To better evaluate the proposed approach, it is assumed that the association between the measurements and targets is known to the Kalman filter (only by internal sensors for comparison). Since the transformation between local coordinate and global coordinate is unknown, observations from external sensor are not used (It is possible to estimate the location and orientation of the external sensor, when data association is given or calculated by both the internal and the external sensors. Once the transformation between two coordinate systems is confirmed, it is expected that the Kalman filter should be the optimal filter for the localization task. This is out of the scope of this paper as we only consider that the data association and the corresponding transformation in unknown environments).

The PHD filter also avoids the data association challenges under the Random Finite Set (RFS) statistics, but only measurements from internal sensors are used to estimate the states ( it is still impossible to utilize measurements from external sensor with unknown configuration).

Assume each measurement $\left(p_{x}, p_{y}\right)$ takes one communication bit in the network communication, for both the internal and external sensors. Then SME filter requires only $2 N$ bits bandwidth at each step, which also meets the minimal requirements for the network communicating (There is a total of $N$ measurements acquired by external sensor and $N$ measurements acquired by internal sensors. To communicate the whole measurement on the network, $2 N$ bits bandwidth is thus the minimal requirement. In contrast to decentralized solutions, the SME filter only operates on the measurements level whereas the others operate on both the states and covariances level).

Figure 2 exhibits true trajectories and the corresponding estimations in three scenarios: one CV scenario and two CTM scenarios (two and four vehicles are maneuvering in CTM scenarios, with $\omega= \pm 0.05^{\circ}$ ). Although Kalman filter has been proved as the optimal Bayesian filter in linear environment, the corresponding precisions are worse than the SME filter. Also compared to the PHD filter, the SME filter performs better.

The high performance of the SME filter is mainly from the topology information measured by the external sensor. Although the external sensor's configuration is unknown, the topology information is still utilized. This is visualized in Fig. 1. It is observed that either in global or in local coordinate system, the distances between vehicles are invariant. In Kalman filter, it is not possible to use this data from external sensor. Still, the performance of the Kalman filter is better than the PHD filter, which is due to the fact that the PHD calculations are based on prop- 


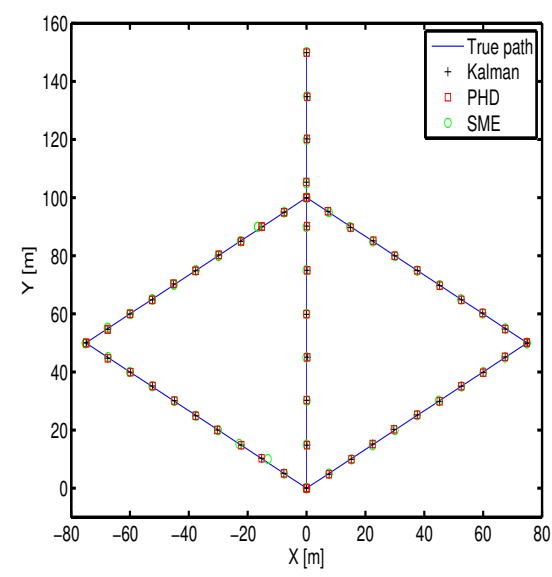

(a) CV model

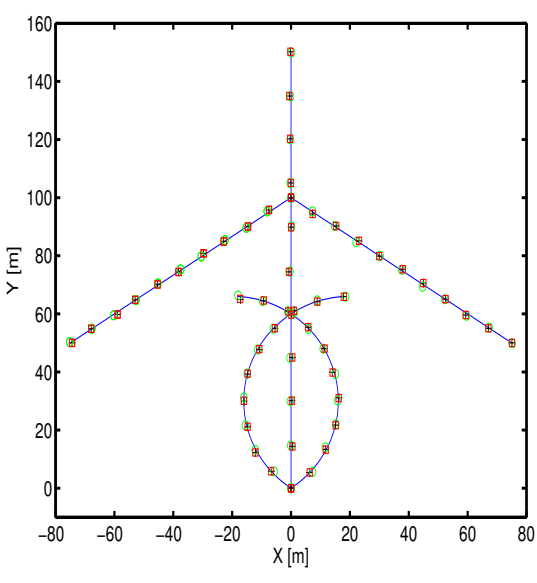

(b) CTM model

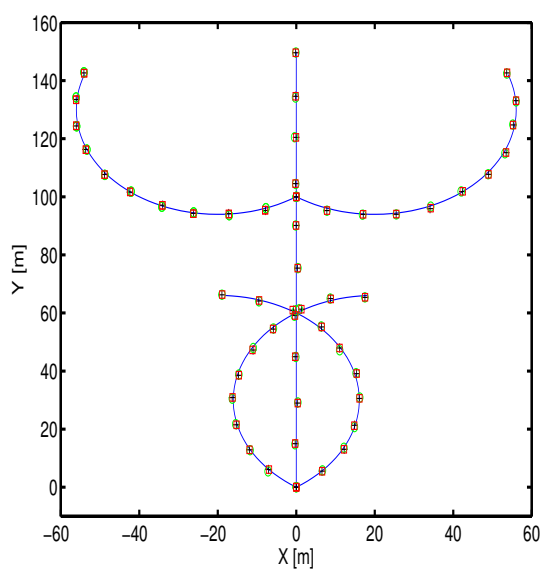

(c) CTM model

Figure 2: Vehicles' true trajectories and the estimations 


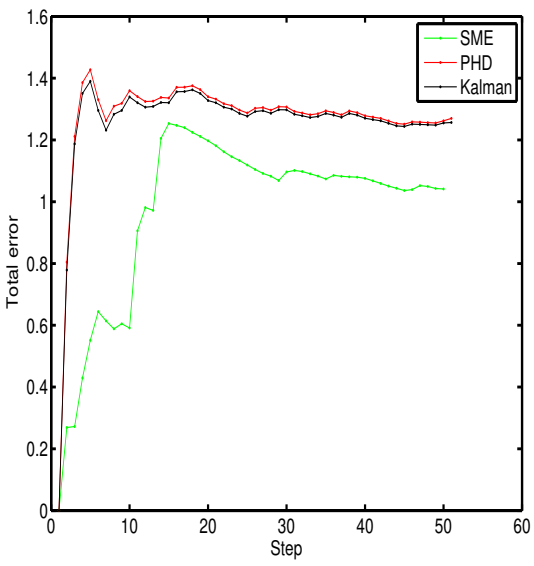

(a) CV model

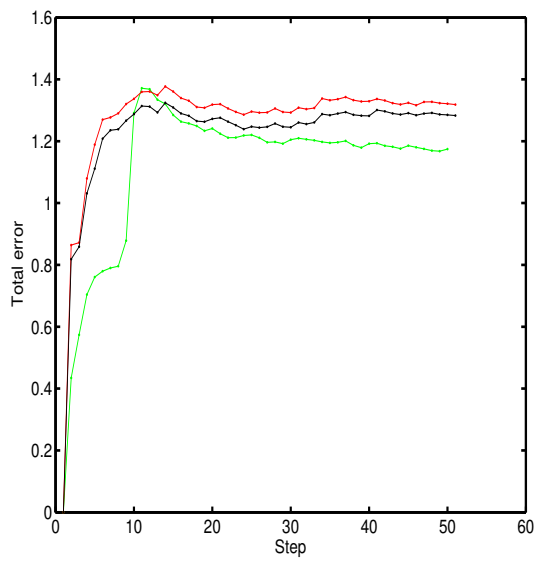

(b) CTM model with two maneuver vehicles

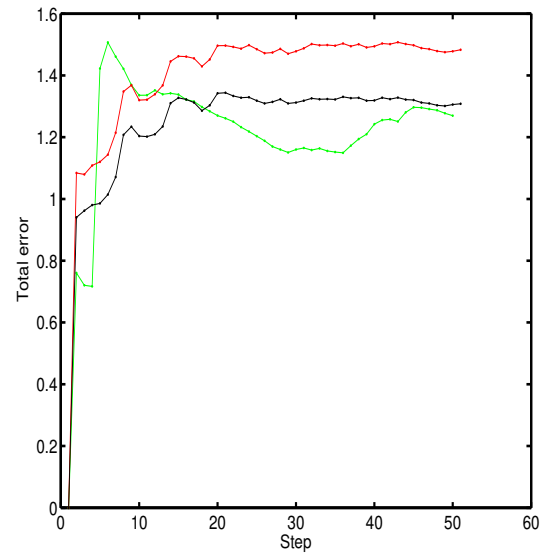

(c) CTM model with four maneuver vehicles

Figure 3: The performance of the estimation 
agation of a multi-target first-order moment statistic, whereas Kalman filter uses each targets' posterior distribution.

Figure 3 indicates the precision of all methods by calculating the RMSE (root mean square deviation) value. The total error is calculated by summing up the RMSE of all vehicles as follows:

$$
\begin{gathered}
\text { Error }=\left(p_{x}^{\text {est }}-p_{x}^{\text {true }}\right)^{2}+\left(p_{y}^{e s t}-p_{y}^{\text {true }}\right)^{2} \\
\text { Total_error }=\sqrt{\frac{\sum_{j=1}^{n} \sum_{i=1}^{N} \text { Error }_{i}^{j}}{n}}
\end{gathered}
$$

where $n$ is the step index (The PHD filter estimates the vehicles as a set-valued state, it does not distinguish them. Individual states are unordered within the setvalued state. To compare the performance, a state-to-target association process is implemented to label the PHD estimations).

Fig. 3 illustrates that each filter has a certain estimation error which depends on each sensors' capability. This error is caused by the uncertainties in the measurements; as no errant data was introduced to the system, these values should represent the lower bound of the estimate. It shows that the overall performance of the SME filter is better than the others.

Although the SME filter utilizes additional precise measurements, the improvement still does not match the lower bound of the external sensor. This is because the topology information only represents the distributions among vehicles, which partly represents the localization information. In contrast to the scenario when the coordinate transformation is known, the external sensor provides the complete information and there should be an improvement in the estimation.

Fig. 3 also illustrates that the performance of the SME filter depends on the vehicles' behaviors. It is observed that for CTM with $\omega= \pm 0.05^{\circ}$ for two vehicles, the performance of the SME filter is almost same as the CV scenario. For CTM with $\omega= \pm 0.05^{\circ}$ for four vehicles, the performance degrades a lot when compared to $\mathrm{CV}$ scenario. This results are similar for the PHD filter. This phenomenon is explained as follows:

In Kalman filter, each vehicle relies on its own measurements during the filtering phase. The precision of the estimation relies on the uncertainties of its own measurements. On the other hand, in SME filter, the state is estimated by the pseudo-measurements which consist of all the measurements. If some measurements have unexpected noises from vehicles, the influence on the pseudomeasurement space is negligible. This phenomenon helps the CV scenario, but for 
CTM scenarios SME filter may consider various maneuver of vehicles as noises from sensors. With the additional information from the external sensor, the performance of the SME filter is thus ensured. The PHD filter treats the measurements as set valued measurement but without considering any information from external sensor. This does not impact the performance of CV scenario but for the CTM it degrades.

\subsection{Discussion}

To study the robustness of the SME filter in real applications, the following issues need further discussion.

\subsubsection{False detection and missed detection}

In this paper, both internal and external sensors are assumed to work in an ideal environment which means that all the measurements originate from the vehicles. The SME filter estimates the states without considering the data association issue. However, in practice, the number of measurements $M$ may not be equal to the number of vehicles $\mathrm{N}$, which can be caused by false or missed detections (due to clutter). In order to address this challenge, the SME filter should be implemented in parallel, c.p. [26], [27]. These specific details are not the focus of this paper.

\subsubsection{External sensor estimation}

To jointly estimate the vehicles and the infrastructure configuration, the transformation between the coordinates needs to be calculated. But there are two major problems: the data association problem and the over-convergence problem. As the key contribution of the proposed paper is to localize vehicles in an unknown data association scenario, coordinates transformation is thus not considered. Overconvergence problem is due to the stochastic interdependence between the estimations when sharing the information [4]. For example, a scenario when the configuration of the external sensor has already been estimated. The observation from external sensor cannot be directly used since the transformed information is dependent to the internal sensors.

\subsubsection{Implementation issue}

Since the SME filter transforms the original measurements from linear space to the non-linear space by using the symmetric measurement equations, the corresponding targets' states are thus estimated based on the non-linear Bayesian filters, e.g. Extended Kalman filter, Unscented Kalman filter and the particle filter. In this paper, five vehicles polynomials up to order five are considered. Due to the highly 
nonlinear functions in such situation, only the particle filter is utilized. Both the EKF and UKF do not give satisfying results due to the strong non-linearities and numerical instabilities.

It should be noticed that due to the non-linear transformation, the symmetric transformations are unsuitable for larger target numbers as the order of the involved polynomial increases with the amounts, i.e., for 10 vehicles polynomials up to order 10 are required. Therefore, for large number of vehicles, the SME filter do not give satisfying results due to the high nonlinearities.

\section{- Benefits}

First, data association is avoided. With the symmetric measurement equations, the data association issue is traded for another difficult, but different, problem. Thus the SME filter only focuses on the analytic nonlinear estimation. By utilizing the homogeneous symmetric functions, the original measurements are projected to the pseudo-measurements where no information is lost during the transformation. In this way, it is possible to estimate states without considering the association between measurements and targets.

Second, the requirement for communication bandwidth is minimized. The inter-communication system only transmits the original measurements to the SME filter which results in minimal consumption requirements. In contrast to decentralized solutions, it does not rely on each vehicle's state and the corresponding covariance in fusion process.

Third, coordinate transformation is not required. By using the topology information among vehicles, the coordinate transformation is avoided. Even if the configuration of the infrastructure is unknown, measurements can still be utilized by the SME filter.

\section{Conclusion}

In this paper, a recursive Bayesian solution for vehicle-infrastructure cooperative localization is proposed. The measurement-to-track association uncertainty, communication bandwidth issue, and unknown coordinates transformation problem make cooperative localization complex and infeasible. A SME filter solution is proposed to address all of the mentioned issues simultaneously. In comparison to the related work, all vehicles are considered as a joint single state which is updated with the symmetric measurement equations. The proposed method has been evaluated in simulations and the results demonstrate precision which is 
proportional to the Kalman Filter, but with improved efficiency and without the requirement for known data association. To fully demonstrate the applicability of this algorithm in complex car-to-car and car-to-infrastructure scenarios, further consideration should be paid to clutter, obscuration and erroneous measurements.

\section{ACKNOWLEDGMENTS}

This work is partially supported by the SADA project funded by the German ministry of economics (BMWi), within the program 'IKT für Elektromobilität III'.

[1] F. Tian, C. Wu, D. Chu, C. Sun, T. Zhou, Experimental design of integrated platform for demonstration of cooperative vehicle infrastructure systems in china, in: Intelligent Transportation Systems (ITSC), 2014 IEEE 17th International Conference on, 2014, pp. 105-108. doi:10.1109/ITSC.2014.6957674.

[2] N. Boasman, D. Clarke, S. Davison, R. Stokes, Advanced test methods for integrated navigation systems, Royal Institute of Navigation.

[3] J. Laneurit, R. Chapuis, F. Chausse, Accurate vehicle positioning on a numerical map, International Journal of Control, Automation, and Systems 3 (1) (2005) 15-31.

[4] F. Zhang, H. Staehle, G. Chen, C. Buckl, A. Knoll, Multiple vehicle cooperative localization under random finite set framework, in: IEEE/RSJ International Conference on Intelligent Robots and Systems (IROS), 2013.

[5] K. chan Lan, C.-M. Huang, C.-Z. Tsai, On the locality of vehicle movement for vehicle-infrastructure communication, in: ITS Telecommunications, 2008. ITST 2008. 8th International Conference on, 2008, pp. 116-120. doi:10.1109/ITST.2008.4740240.

[6] S. Roumeliotis, I. Rekleitis, Analysis of multirobot localization uncertainty propagation, in: Intelligent Robots and Systems, 2003. (IROS 2003). Proceedings. 2003 IEEE/RSJ International Conference on, Vol. 2, 2003, pp. 1763 - 1770 vol.2. doi:10.1109/IROS.2003.1248899.

[7] D. Fox, W. Burgard, S. Thrun, Markov localization for mobile robots in dynamics environments, Journal of Artificial Intelligence Research 11 (1999) 391-427. 
[8] A. Howard, M. Matark, G. Sukhatme, Localization for mobile robot teams using maximum likelihood estimation, in: Intelligent Robots and Systems, 2002. IEEE/RSJ International Conference on, Vol. 1, 2002, pp. 434 - 439 vol.1. doi:10.1109/IRDS.2002.1041428.

[9] E. Nerurkar, S. Roumeliotis, A. Martinelli, Distributed maximum a posteriori estimation for multi-robot cooperative localization, in: Robotics and Automation, 2009. ICRA '09. IEEE International Conference on, 2009, pp. 1402 -1409. doi:10.1109/ROBOT.2009.5152398.

[10] E. Kamen, Multiple target tracking based on symmetric measurement equations, Automatic Control, IEEE Transactions on 37 (3) (1992) 371-374. doi:10.1109/9.119640.

[11] F. Zhang, G. Hinz, D. Clarke, A. Knoll, Vehicle-infrastructure localization based on the sme filter, in: Connected Vehicles and Cooperative Systems Workshop, Intelligent Transportation Systems (ITSC), 2015 IEEE 18th International Conference on, 2015.

[12] Swati, S. Bhaumik, Multiple target tracking using homogeneous symmetric measurement, in: Recent Advances in Information Technology (RAIT), 2012 1st International Conference on, 2012, pp. 707-712. doi:10.1109/RAIT.2012.6194581.

[13] E. Kamen, C. Sastry, Multiple target tracking using products of position measurements, Aerospace and Electronic Systems, IEEE Transactions on 29 (2) (1993) 476-493. doi:10.1109/7.210085.

[14] N. Karam, F. Chausse, R. Aufrere, R. Chapuis, Cooperative multi-vehicle localization, in: Intelligent Vehicles Symposium, 2006 IEEE, 2006, pp. 564 -570. doi:10.1109/IVS.2006.1689688.

[15] H. Li, F. Nashashibi, Cooperative multi-vehicle localization using split covariance intersection filter, in: Intelligent Vehicles Symposium (IV), 2012 IEEE, 2012, pp. 211 -216. doi:10.1109/IVS.2012.6232155.

[16] S. Roumeliotis, G. Bekey, Distributed multirobot localization, Robotics and Automation, IEEE Transactions on 18 (5) (2002) 781 - 795. doi:10.1109/TRA.2002.803461. 
[17] N. Karam, F. Chausse, R. Aufrere, R. Chapuis, Localization of a group of communicating vehicles by state exchange, in: Intelligent Robots and Systems, 2006 IEEE/RSJ International Conference on, 2006, pp. 519 -524. doi:10.1109/IROS.2006.282028.

[18] SADA, Smart Adaptive Data Aggregation, BMWi, IKT fur Elektromobilitat III (2015).

URL http: / /www.projekt-sada.de

[19] T. E. Fortmann, Y. Bar-Shalom, M. Scheffe, Sonar tracking of multiple targets using joint probabilistic data association, Oceanic Engineering, IEEE Journal of 8 (3) (1983) 173-184. doi:10.1109/JOE.1983.1145560.

[20] R. Mahler, Multitarget bayes filtering via first-order multitarget moments, IEEE Transactions on Aerospace and Electronic Systems (4) (2003) 1152 1178, Vol. 39. doi:10.1109/TAES.2003.1261119.

[21] E. Giannopoulos, R. Streit, P. Swaszek, Probabilistic multi-hypothesis tracking in a multi-sensor, multi-target environment, in: Data Fusion Symposium, 1996. ADFS '96., First Australian, 1996, pp. 184-189. doi:10.1109/ADFS.1996.581104.

[22] W. Leven, A. Lanterman, Unscented kalman filters for multiple target tracking with symmetric measurement equations, Automatic Control, IEEE Transactions on 54 (2) (2009) 370-375. doi:10.1109/TAC.2008.2008327.

[23] L. W.F., L. A.D., Multiple target tracking with symmetric measurement equations using unscented kalman and particle filters, in: System Theory, 2004. Proceedings of the Thirty-Sixth Southeastern Symposium on, 2004, pp. 195-199. doi:10.1109/SSST.2004.1295647.

[24] M. Baum, U. Hanebeck, The kernel-sme filter for multiple target tracking, in: Information Fusion (FUSION), 2013 16th International Conference on, 2013, pp. 288-295.

[25] X. Li, V. Jilkov, Survey of maneuvering target tracking. part i. dynamic models, Aerospace and Electronic Systems, IEEE Transactions on 39 (4) (2003) 1333-1364. doi:10.1109/TAES.2003.1261132.

[26] F. E. Daum, Cramer-rao bound for multiple-target tracking, in: Proc. SPIE, Vol. 1481, 1991, pp. 341-344. doi:10.1117/12.45667. 
[27] E. W. Kamen, Y. J. Lee, C. R. Sastry, Parallel sme filter for tracking multiple targets in three dimensions, in: Proc. SPIE, Vol. 2235, 1994, pp. 417-428. doi:10.1117/12.179068. 